\title{
Formation of the $\Delta^{18,19}$ Double Bond and Bis(spiroacetal) in Salinomycin Is Atypically Catalyzed by SInM, a Methyltransferase-like Enzyme**
}

\author{
Chunyan Jiang, Zhen Qi, Qianjin Kang, Jing Liu, Ming Jiang, and Linquan Bai*
}

\begin{abstract}
Salinomycin is a widely used polyether coccidiostat and was recently found to have antitumor activities. However, the mechanism of its biosynthesis remained largely speculative until now. Reported herein is the identification of an unprecedented function of SInM, homologous to O-methyltransferases, by correlating its activity with the formation of the $\Delta^{18,19}$ double bond and bis(spiroacetal). Detailed in vivo and in vitro investigations revealed that $\operatorname{SIn} M$, using positively charged $S$-adenosylmethionine (SAM) or sinefungin as the cofactor, catalyzed the spirocyclization-coupled dehydration of C19 in a highly atypical fashion to yield salinomycin.
\end{abstract}

P olyether ionophores, a structurally unique group of natural products, have the ability to chelate metal ions selectively within a hydrophilic matrix and then transport the ions across cell membranes, thus leading to depolarization and succedent cell death..$^{[1]}$ This group of antibiotics are widely used as anticoccidial agents and growth promoters in animal husbandry. ${ }^{[2]}$ Salinomycin (Figure 1), isolated from Streptomyces albus, is an important commercial polyether antibiotic with a complex bis(spiroacetal) core structure. Recent reports on the outstanding potency to selectively kill cancer stem cells have led to considerable interest in its biosynthetic mechanism. ${ }^{[3]}$

Our group ${ }^{[4]}$ as well as that of Leadlay ${ }^{[5]}$ have cloned and characterized the gene clusters responsible for the biosyn-

[*] Dr. C. Jiang, ${ }^{[+]}$Z. Qi ${ }^{[+]}$Dr. Q. Kang, Dr. M. Jiang, Prof. Dr. L. Bai

State Key Laboratory of Microbial Metabolism

School of Life Sciences \& Biotechnology

Shanghai Jiao Tong University

800 Dongchuan Rd. Shanghai 200240 (China)

E-mail: bailq@sjtu.edu.cn

J. Liu

Institute of Health Sciences

School of Chemistry and Chemical Engineering

Anhui University, Hefei 230601 (China)

$\left.{ }^{+}\right]$These authors contributed equally to this work.

[**] This work was supported by the Ministry of Science and Technology of China $(2012$ CB721005, 2012AA022107) and the National Natural Science Foundation of China (31400030, 31470157). We kindly thank Prof. David E. Cane (Brown University) and Prof. Yuemao Shen (Shandong University) for helpful discussions.

Supporting information for this article is available on the WWW under http://dx.doi.org/10.1002/anie.201503561.

(C) 2015 The Authors. Published by Wiley-VCH Verlag GmbH \& Co. $\mathrm{KGaA}$. This is an open access article under the terms of the Creative Commons Attribution Non-Commercial License, which permits use, distribution and reproduction in any medium, provided the original work is properly cited and is not used for commercial purposes.
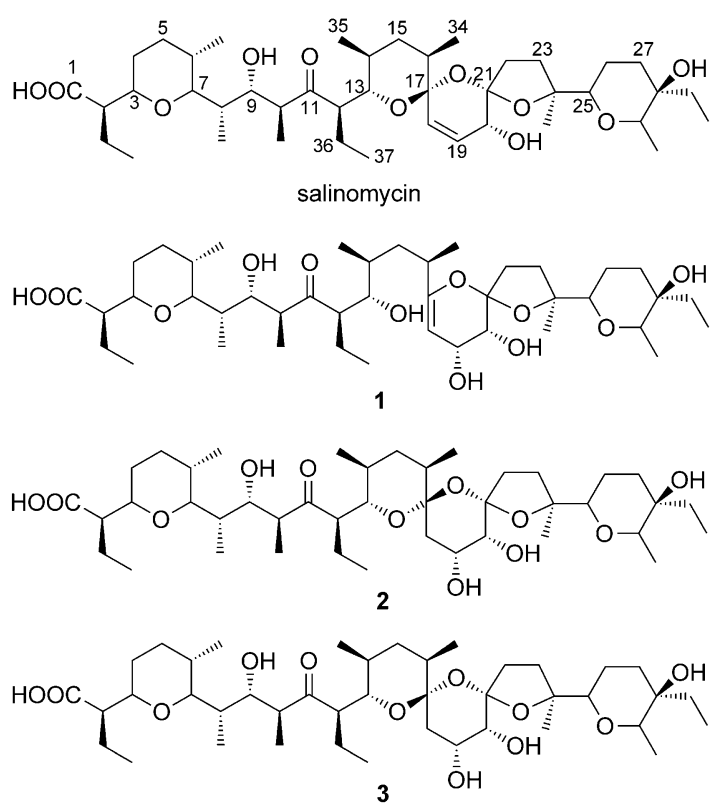

Figure 1. Chemical structures of salinomycin and the compounds 1, 2, and 3.

thesis of salinomycin from S. albus XM211 and DSM 41398, respectively. Salinomycin is assembled by a typical type I polyketide synthase (PKS). In the majority of cases, the double-bond formation in the polyketides is directly attributed to the presence of a ketoreductase (KR) and dehydratase $(\mathrm{DH})$ di-domain in the appropriate module. ${ }^{[6]}$ Occasionally, formations of the unsaturated moieties are dependent upon post-PKS enzymes, instead of a canonical DH domain. ${ }^{[7]}$ In the PKS assembly line of salinomycin, modules 1 and 3 contain the expected KR-DH di-domains responsible for the double-bond formation of $\Delta^{28,29}$ and $\Delta^{24,25}$, which serve as targets for the subsequent epoxidation and epoxide-opening cascade ${ }^{[8]}$ Module 6 has a KS-AT-KR-ACP domain structure but lacks a DH domain for the required dehydration, as deduced from the presence of a double bond between C18 and C19 in salinomycin. Similarly, module 14 also lacks a DH domain for the putative double-bond formation between $\mathrm{C} 2$ and $\mathrm{C} 3$, and subsequent formation of the tetrahydropyran ring. These discrepancies suggest the possibility that these two double bonds may be installed by post-PKS tailoring steps. To identify the required enzymes for the installation of double bonds $\Delta^{18,19}$ and $\Delta^{2,3}$, we examined the biosynthetic gene cluster of salinomycin for likely candidates. However, no putative dehydratase gene has been found in the gene cluster. 
Until now, the mechanisms of the double-bond formation have remained obscure.

Within the gene cluster of salinomycin, immediately downstream of the type II thioesterase gene $(\sin D I)$ resides $\sin M$. In silico analysis revealed that SlnM shows moderate homology to TcmP in the tetracenomycin biosynthesis $(37 \%$ identity, $59 \%$ similarity), and catalyzes methylation of a carboxy group. ${ }^{[9]}$ Moreover, the amino-acid sequence of SlnM contains a glycine-rich motif, LGTGLDG, at the $\mathrm{N}$ terminus (see Figure S1 in the Supporting Information), similar to a signature motif for SAM binding (GXGXG) ${ }^{[10]}$ The homology to the carboxy methyltransferase suggested that this enzyme might act as a SAM-dependent methyltransferase in the biosynthesis of salinomycin. However, there is no methylated salinomycin detected by HPLC-MS analysis of $S$. albus XM211 fermentation extracts.

To investigate the putative involvement of $\operatorname{Sin} M$ in the biosynthesis of salinomycin, the gene $\sin M$ was inactivated in S. albus XM211. ${ }^{[4]}$ HPLC analysis showed that the resulting mutant JCY38 lost the ability to produce salinomycin, but instead accumulated 1, a new and more hydrophilic compound (see Figure S4). Further complementation of JCY38 by the cloned $\sin M$ gene restored the production of salinomycin, and confirmed that the loss of salinomycin productivity and the accumulation of $\mathbf{1}$ were caused by the replacement of $\sin M$. The compound $\mathbf{1}$ was further isolated from the liquid culture of JCY38 and characterized by NMR spectroscopy (see the Supporting Information). The molecular formula of $\mathbf{1}$ was determined to be $\mathrm{C}_{42} \mathrm{H}_{72} \mathrm{O}_{12}$, which has an increase by 18 in the molecular weight relative to salinomycin. Interpretation of the NMR spectroscopic data revealed that the presence of a $\Delta^{17,18}$ double bond, which was supported by the HMBC correlations from $\mathrm{H} 16$ to $\mathrm{C} 14, \mathrm{C} 15, \mathrm{C} 17, \mathrm{C} 18$, and $\mathrm{C} 34$, and from $\mathrm{H} 18$ to $\mathrm{C} 16, \mathrm{C} 17, \mathrm{C} 19$, and $\mathrm{C} 20$. Moreover, HMBC correlations from $\mathrm{H} 19$ to $\mathrm{C} 17$, $\mathrm{C} 18, \mathrm{C} 20$, and $\mathrm{C} 21$ indicate the presence of a hydroxy group at $\mathrm{C} 19$, and was further confirmed by the correlation between $\mathrm{H} 19$ and $\mathrm{H} 18 / \mathrm{H} 20$ in a ${ }^{1} \mathrm{H}-{ }^{1} \mathrm{H}$ COSY experiment. The presence of a $\mathrm{C} 19$ hydroxy group is consistent with the KSAT-KR-ACP domain structure of module 6 of sal PKS. Furthermore, the HMBC correlations from $\mathrm{H} 13$ to $\mathrm{C} 12$, $\mathrm{C} 14, \mathrm{C} 15, \mathrm{C} 35$, and C36 intriguingly identified a hydroxy group at $\mathrm{C} 13$ (Figure 1).

In addition, LC-MS analysis of the $\Delta \sin M$ mutant JCY38 revealed the accumulation of two other compounds, $\mathbf{2}$ and $\mathbf{3}$, with the same mass as $\mathbf{1}\left(\mathrm{m} / \mathrm{z}, 791.5[M+\mathrm{Na}]^{+}\right)$but different retention times (see Figure S5), and they were purified and subjected to ${ }^{1} \mathrm{H}$ NMR spectroscopic analysis. According to LC-MS (see Figures S6 and S7) and ${ }^{1} \mathrm{H}$ NMR analyses (see the Supporting Information), $\mathbf{2}$ and $\mathbf{3}$ (Figure 1) were proven to be most likely identical to the previously reported analogues of salinomycin in S. albus DSM 41398 (DsalE). ${ }^{[1]}$ Although all of the three compounds shared the same molecular formula, 1 displays a significant UV absorption at $\lambda=210 \mathrm{~nm}$ and could be detected easily by HPLC, whereas no obvious absorption peak for either $\mathbf{2}$ or $\mathbf{3}$ was observed in the UV/Vis spectra. During the purification process, $\mathbf{1}$ was found to be slowly converted into $\mathbf{2}, \mathbf{3}$, and other minor compounds (see Figure S8).
The accumulation of $\mathbf{1 , 2}$, and $\mathbf{3}$ in a $\Delta \sin M$ mutant JCY38 suggested that $\operatorname{SinM}$ is a post-PKS tailoring enzyme in the biosynthesis of salinomycin. Characterization of its function was firstly performed by feeding the crude extract of fermentation broth of JCY38 to the culture of JCY34 (a $\Delta \sin A 9$ PKS mutant), in which the assembly of the polyketide backbone of salinomycin is abolished and the $\sin M$ gene remains intact. As a result of a new peak, having the same retention time and molecular mass as those of the authentic standard of salinomycin, was detected by LC-MS and Q-TOFMS (see Figures S9 and S10). This result indicated that SInM could transform the products of the $\Delta \sin M$ mutant into salinomycin in vivo. To further ascertain the real substrate, $\mathrm{S} \ln \mathrm{M}$ was over-expressed as an $\mathrm{N}$-terminal glutathione- $S$ transferase (GST) fusion protein in E. coli BL21(DE3) (see Figure S13). We incubated a mixture of $\mathbf{1}, \mathbf{2}$, and $\mathbf{3}$ with the cell-free extract of the recombinant E. coli strain, and performed a time-course analysis of product accumulation and substrate consumption. Indeed, salinomycin was only generated in the presence of GST-SlnM in a relatively rapid, time-dependent manner, while 1 decreased accordingly. Under these enzymatic reaction conditions, no conversion of either $\mathbf{2}$ or $\mathbf{3}$ into salinomycin was observed (Figure 2).

SlnM exhibits moderate sequence similarity to methyltransferases and contains conserved residues for SAM binding, thus suggesting that SAM might be the substrate donating a methyl group for the SinM-catalyzed enzymatic reaction. As expected, incubation of GST-SInM with $\mathbf{1}$ in the presence of SAM generated salinomycin, whereas no reaction occurred when either SAM was omitted or $S$-adenosyl-L-homocysteine (SAH) was added to the reaction mixture (Figure 3). Then, the activity of $\operatorname{SinM}$ at various temperatures and $\mathrm{pH}$ values was measured. Purified GST-SInM was optimally active at $35^{\circ} \mathrm{C}$ and a $\mathrm{pH}$ value of 7.0-7.5 (see Figure S14). The $K_{\mathrm{M}}$ and

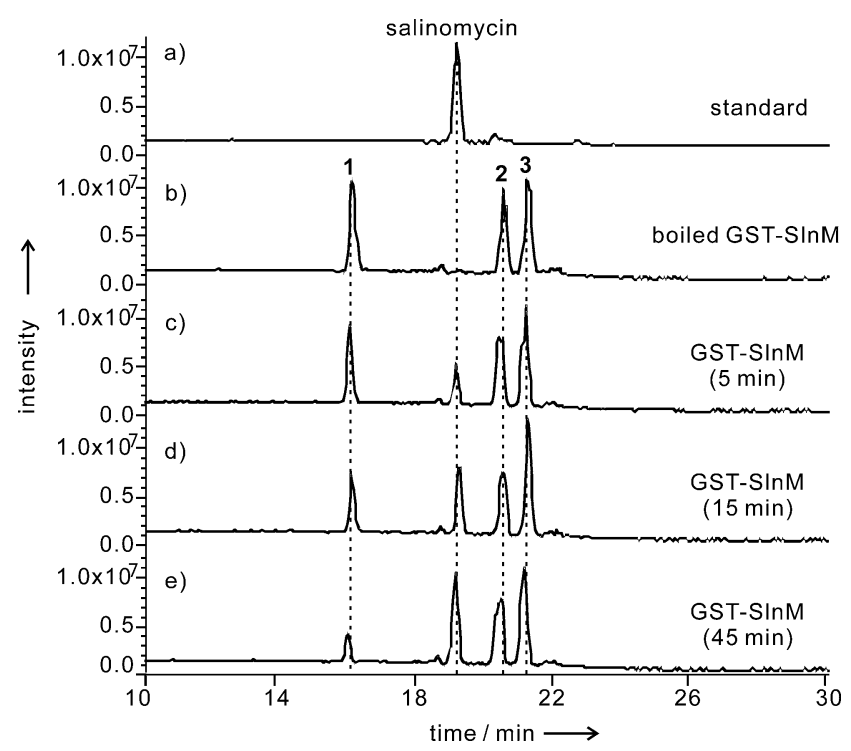

Figure 2. HPLC-MS analysis of in vitro assays catalyzed by cell-free extract of E. coli expressing GST-SInM. The mixture of 1, 2, and $\mathbf{3}$ was incubated with the cell-free extract of E. coli expressing GST-SInM for $5 \mathrm{~min}$ (c), $15 \mathrm{~min}$ (d), and $45 \mathrm{~min}$ (e). Trace (b) is the negative control using boiled cell-free extract with denatured GST-SInM. 


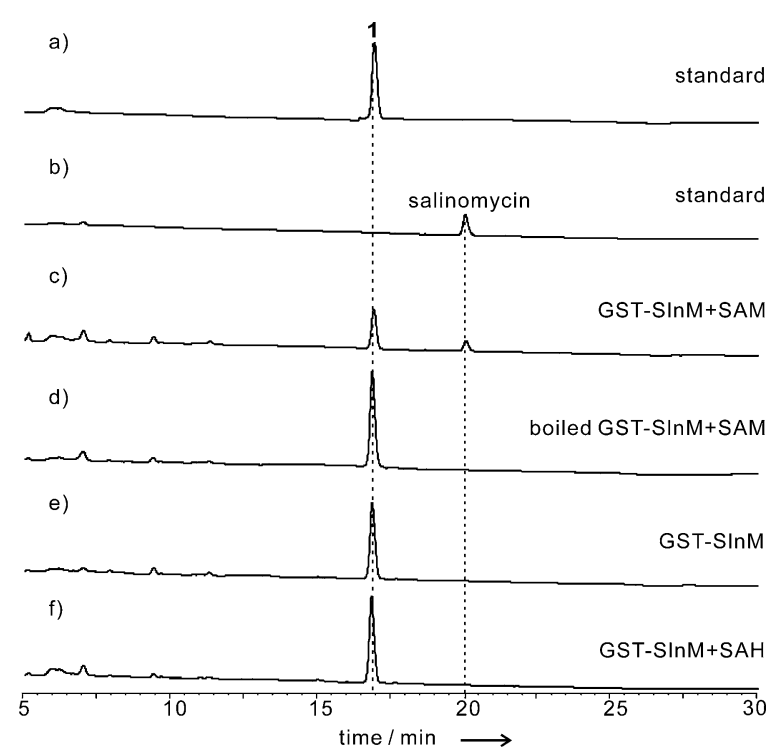

Figure 3. HPLC analysis of assays catalyzed by SInM. Assay reactions were incubated at $35^{\circ} \mathrm{C}$ in the presence of purified GST-SInM with SAM (c), SAH (f), or without SAM/SAH (e). Heat-denatured GSTSInM was used as a negative control (d).

$k_{\text {cat }}$ values of $\mathbf{1}$ for the formation of salinomycin were (17.7 \pm 4.9) $\mu \mathrm{M}$ and $(1.50 \pm 0.12) \mathrm{min}^{-1}$, respectively, thus giving a $k_{\text {cat }} /$ $K_{\mathrm{M}}$ of $0.084 \mu \mathrm{M}^{-1} \mathrm{~min}^{-1}$ (see Figure S15).

Thus far, both in vivo and in vitro experiments validated that in the presence of SAM, SInM catalyzed a post-tailoring process, thus leading the conversion of $\mathbf{1}$ into salinomycin. However, when we performed a time-course analysis of SAM consumption, HPLC analysis showed neither consumption of SAM nor accumulation of SAH in the reaction mixture (see Figure $4 \mathrm{a}$ and Figure S16). Additionally, SAH showed no significant inhibition to the catalytic activity of SlnM, even at a high concentration (20-fold of SAM; Figure $4 \mathrm{~b}$ ), whereas most SAM-dependent methylation reactions are strongly inhibited by $\mathrm{SAH} \cdot{ }^{[12]}$ Furthermore, another methyltransferase inhibitor, sinefungin, a SAM analogue which is unable to donate a methyl group ${ }^{[13]}$ was found to activate the activity of SinM, comparable to that of SAM (Figure $4 \mathrm{c}$ ). Comparison between SAM, sinefungin, and SAH reveals that the sulfur of SAM and the $\varepsilon$-amino group of sinefungin carry a formal positive charge at physiological $\mathrm{pH}$, whereas $\mathrm{SAH}$ is uncharged at the corresponding position. Therefore, SAM could be substituted by sinefungin but not SAH as a cofactor in the reaction, indicating that the positive charges of SAM and sinefungin are crucial for the enzymatic catalysis.

A mechanism for the catalysis by $\operatorname{Sin} M$ is proposed in Scheme 1. The attack of the C13 hydroxy group on C17 in 1 involves a $S_{N}$ displacement of the $\mathrm{C} 19$ allylic hydroxy group. Most likely, the hydroxy enol ether of $\mathbf{1}$ is converted into the corresponding cyclohexenone by acid-catalyzed dehydration initiated by protonation of the C19 hydroxy and loss of water. Unlike a proton or a Bronsted acid, each of which has a vacant orbital, the sulfonium of SAM has a fully occupied valence shell and cannot directly interact with the lone pair of the C19 hydroxy group of $\mathbf{1}$. We speculate that SAM or a)

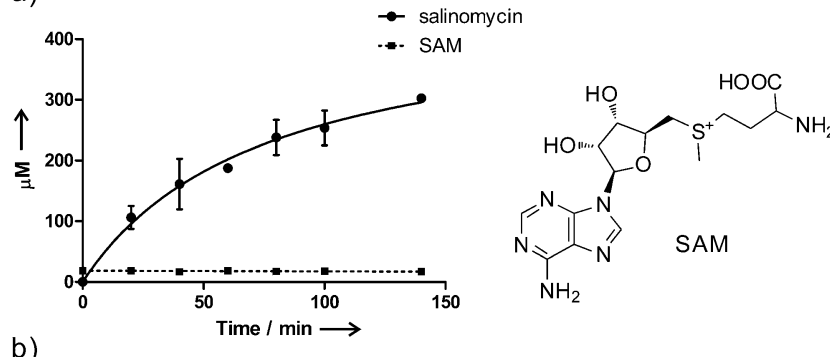

b)
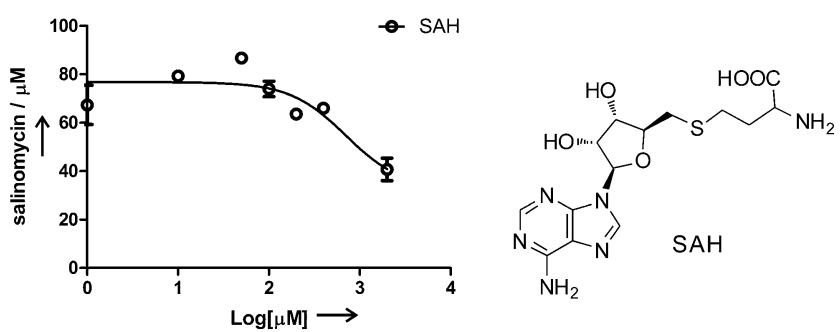

c)

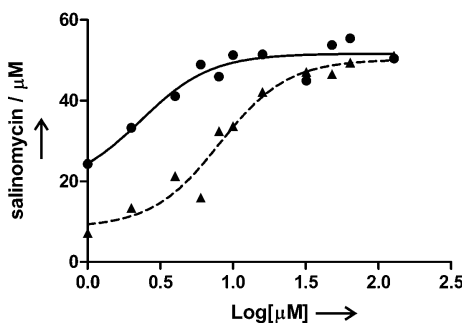

- SAM

-2. sinefungin

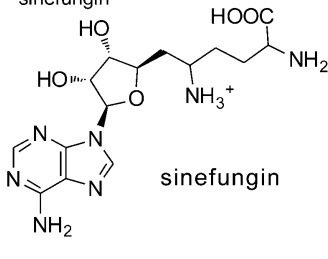

Figure 4. Identification of cofactors for the SInM-catalyzed reaction. a) Detection of the concentration changes of salinomycin and SAM in reactions with varied incubation times (from 0 to $140 \mathrm{~min}$ ). Reactions were conducted with $10 \mu \mathrm{M}$ GST-SInM and $20 \mu \mathrm{M}$ SAM, terminated at $20,40,60,80,100$, or 140 min and quantified by HPLC. b) Inhibition of SInM activity by SAH in the assays with $20 \mu \mathrm{M}$ SAM. c) Activation of SInM activity by SAM or sinefungin. See the Supporting Information for reaction conditions.
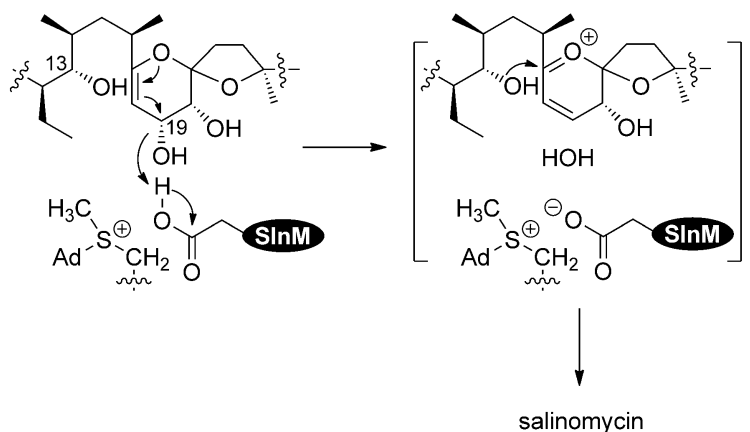

Scheme 1. Proposed mechanism for the reaction catalyzed by SInM.

sinefungin not only stabilize SlnM in an active conformation, but also interact with the actual active site acid to increase its acidity. To the best of our knowledge, this is the first example of a methyltransferase-like enzyme catalyzing the formation of a double bond and bis(spiroacetal), and is reminiscent of the aclacinomycin 10-hydroxylase RdmB, which catalyzes a SAM-dependent hydroxylation step in the biosynthesis of $\beta$-rhodomycin. It has been proposed that the positive charge 
of SAM might facilitate the delocalization of electrons into the anthraquinone core of the substrate. ${ }^{[14]}$

We postulated that some acidic amino-acid residues might act as a base catalyst for protonation of the hydroxy group at C19. Computational modeling of SlnM was performed using I-TASSER. ${ }^{[15]}$ SlnM appears to possess a Rossmann-like methyltransferase fold similar to that of the the template from Mycobacterium leprae ${ }^{[10 \mathrm{~b}, 16]}$ albeit they share very limited sequence similarity. In the modeled SInM-SAM complex, ten residues with acidic side chains (E13, D48, D58, H87, D93, D111, E156, E165, E166, and D186) were found to be located within a short distance from the sulfur group of SAM (see Figure S17). To identify the potential catalytic base, they were mutated to alanine or other amino acids and subsequently overproduced in $E$. coli BL21(DE3). In the enzymatic reactions with cell-free extracts of each mutated SlnM, 1, and SAM (see Table S4), the formation of salinomycin was still observed with E13A-, D48A-, H87A-, E165A-, and E166A-mutated proteins. SlnM with D93A or E156A mutations lost the catalytic activity, while those with D93N or E156Q mutations retained partial activity, thus implying that D93 and E156 are important in maintaining structural integrity. In the reactions with D58A-, D58N-, D111A-, D111N-, D186A-, and D186N-mutated SlnM proteins, the production of salinomycin was completely abolished. Among these three residues, D111 was found to be strongly conserved in methyltransferases, and has been proven to be involved in the formation of hydrogen bonds to hydroxy groups of the SAM ribose. ${ }^{[10 c]}$ These results suggested that D58 and D186 might be active-site residues essential for catalysis or substrate binding.

It is well-known that the glycine-rich motif GXGXG in SAM-dependent methyltransferases plays an important role in SAM binding. ${ }^{[17]}$ To prove whether the motif $\mathrm{LG}_{89} \mathrm{TG}_{91} \mathrm{LDG}$ in SInM is involved in the biochemical activity of SInM, G91 of SlnM was replaced by alanine in plasmid using site-directed mutagenesis. Compared with the wild-type $\mathrm{N}$-terminal $\mathrm{His}_{6}$-tagged SlnM, the G91A mutant exhibited no activity as detected by Q-TOF-MS analysis (see Table S4), and strongly suggested that this motif is involved in the enzymatic catalysis. The observation is consistent with the previous finding that exogenous SAM was essential for maintaining the activity of SlnM.

In conclusion, the isolation and structure elucidation of $\mathbf{1}$, 2, and $\mathbf{3}$ from the $\Delta \sin M$ mutant and the biochemical characterization of $\operatorname{SinM}$ as a SAM-dependent enzyme provide new insight into the mechanism of the last step in the biosynthesis of salinomycin. Among these three new accumulated compounds, $\mathbf{1}$ can only be efficiently converted by $\operatorname{SinM}$ into salinomycin. Without the enzymatic activity, a small amount of $\mathbf{1}$ can spontaneously undergo spirocyclization to afford $\mathbf{2}$ and $\mathbf{3}$ (see Figure S18). SlnM, as a methyltransferase homologue, utilizes SAM as a co-factor, rather than a substrate, to activate the reaction. SAM, or more specifically its positive charge, has been considered to be involved in the enzymatic conversion, with regard to stabilizing the active conformation of S $\ln M$ and increasing the acidity of the active site as well. Site-directed mutagenesis experiments with SlnM revealed that the residues D58 and D186 are critical for the enzymatic activity, and D91 and D111 are absolutely required for the SAM binding. Thus, SInM was identified as the enzyme responsible for the formation of the $\Delta^{18,19}$ double bond and bis(spiroacetal) in the biosynthesis of salinomycin.

Keywords: biosynthesis - computational chemistry . enzyme catalysis - NMR spectroscopy · polyketides

How to cite: Angew. Chem. Int. Ed. 2015, 54, 9097-9100 Angew. Chem. 2015, 127, 9225-9228

[1] a) F. G. Riddell, Chirality 2002, 14, 121-125; b) A. R. Gallimore, Nat. Prod. Rep. 2009, 26, 266-280.

[2] C. Gumila, M. L. Ancelin, A. M. Delort, G. Jeminet, H. J. Vial, Antimicrob. Agents Chemother. 1997, 41, 523-529.

[3] P. B. Gupta, T. T. Onder, G. Jiang, K. Tao, C. Kuperwasser, R. A. Weinberg, E. S. Lander, Cell 2009, 138, 645-659.

[4] C. Jiang, H. Wang, Q. Kang, J. Liu, L. Bai, Appl. Environ. Microbiol. 2012, 78, 994-1003.

[5] M. E. Yurkovich, P. A. Tyrakis, H. Hong, Y. Sun, M. Samborskyy, K. Kamiya, P. F. Leadlay, ChemBioChem 2012, 13, 66-71.

[6] a) J. Wu, T. J. Zaleski, C. Valenzano, C. Khosla, D. E. Cane, J. Am. Chem. Soc. 2005, 127, 17393-17404; b) R. Reid, M. Piagentini, E. Rodriguez, G. Ashley, N. Viswanathan, J. Carney, D. V. Santi, C. R. Hutchinson, R. McDaniel, Biochemistry 2003, 42, 72-79; c) P. Caffrey, ChemBioChem 2003, 4, 654 657.

[7] a) X. Mo, J. Ma, H. Huang, B. Wang, Y. Song, S. Zhang, C. Zhang, J. Ju, J. Am. Chem. Soc. 2012, 134, 2844-2847; b) N. Palaniappan, M. M. Alhamadsheh, K. A. Reynolds, J. Am. Chem. Soc. 2008, 130, 12236-12237.

[8] D. E. Cane, W. D. Celmer, J. W. Westley, J. Am. Chem. Soc. 1983, 105, 3594-3600.

[9] H. Decker, H. Motamedi, C. R. Hutchinson, J. Bacteriol. 1993, 175, 3876-3886.

[10] a) P. Z. Kozbial, A. R. Mushegian, BMC Struct. Biol. 2005, 5, 19; b) J. L. Martin, F. M. McMillan, Curr. Opin. Struct. Biol. 2002, 12, 783-793; c) D. K. Liscombe, G. V. Louie, J. P. Noel, Nat. Prod. Rep. 2012, 29, 1238-1250.

[11] H. Luhavaya, S. R. Williams, H. Hong, L. Gonzaga de Oliveira, P. F. Leadlay, ChemBioChem 2014, 15, 2081-2085.

[12] a) J. M. Mato, L. Alvarez, P. Ortiz, M. A. Pajares, Pharmacol. Ther. 1997, 73, 265-280; b) A. Bechthold, H. G. Floss, Eur. J. Biochem. 1994, 224, $431-437$.

[13] a) A. J. Kreuzman, J. R. Turner, W. K. Yeh, J. Biol. Chem. 1988, 263, 15626-15633; b) R. T. Borchardt, L. E. Eiden, B. Wu, C. O. Rutledge, Biochem. Biophys. Res. Commun. 1979, 89, 919-924.

[14] a) Y. Wang, J. Niemi, K. Airas, K. Ylihonko, J. Hakala, P. Mantsala, Biochim. Biophys. Acta Protein Struct. Mol. Enzymol. 2000, 1480, 191 -200; b) A. Jansson, J. Niemi, Y. Lindqvist, P. Mantsala, G. Schneider, J. Mol. Biol. 2003, 334, 269-280; c) A. Jansson, H. Koskiniemi, A. Erola, J. Wang, P. Mantsala, G. Schneider, J. Niemi, J. Biol. Chem. 2005, 280, 3636-3644.

[15] a) Y. Zhang, BMC Bioinf. 2008, 9, 40; b) A. Roy, A. Kucukural, Y. Zhang, Nat. Protoc. 2010, 5, 725-738; c) A. Roy, J. Yang, Y. Zhang, Nucleic Acids Res. 2012, 40, W471-477.

[16] M. Graña, A. Haouz, A. Buschiazzo, I. Miras, A. Wehenkel, V. Bondet, W. Shepard, F. Schaeffer, S. T. Cole, P. M. Alzari, Protein Sci. 2007, 16, 1896-1904.

[17] B. P. Pollack, S. V. Kotenko, W. He, L. S. Izotova, B. L. Barnoski, S. Pestka, J. Biol. Chem. 1999, 274, 31531-31542.

Received: April 19, 2015

Published online: June 11, 2015 